\title{
酸性条件下正钛酸钛(IV)中心与过氧化氢反应机理的第一性原理研究
}

\author{
唐㑆 ${ }^{a}$ 陶辉锦*, , , b, d 蒋新宇 ${ }^{c}$ \\ ( ${ }^{a}$ 中南大学材料科学与工程学院 长沙 410083) \\ ( ${ }^{b}$ 中南大学有色金属材料科学与工程教育部重点实验室 长沙 410083) \\ ( ${ }^{c}$ 中南大学化学化工学院 长沙 410083) \\ ( ${ }^{d}$ 中南大学粉末冶金国家重点实验室 长沙 410083)
}

\begin{abstract}
摘要 正钛酸钛(IV)中心与过氧化氢的溶液反应很早就已经开始被加以关注和研究, 因其在化学催化、分析化学以及 精细陶瓷方面具有重要用途. 尽管如此, 其不同条件下的反应机理以及产物长期以来没有得到共识. 本文主要希望通 过对特定条件下 $(\mathrm{pH}<1)$, 对这一反应进行第一性计算研究. 其中计算主要采用 B3LYP 泛函和 6-311G+(3df,2p)基组的 组合, 对可能的反应路径进行分步以及总反应热的计算, 其结果 $(27 \mathrm{~kJ} / \mathrm{mol})$ 与实验值较为接近. 通过仔细研究反应中分 子轨道的变化, 指出成环反应和氢转移反应是整体反应的关键步骤, 采用单向连串反应假设对实验现象进行分析, 并 决定其宏观反应动力学 “准一级” 的特征, 获得了对特定及一般实验条件下的结果的合理解释. 通过对这一反应机理 的研究, 我们希望能够将其推广至其他过渡族金属中心与过氧化物结合的反应中, 得以指导高效环氧化催化剂及精细 陶瓷的合成与制备.
\end{abstract}

关键词 titanium(IV); 过氧化氢; B3LYP; 分子轨道; 反应动力学

\section{Possible Reaction between Titanium(IV) and Hydrogen Peroxide in Acidic Condition: An ab-initio Study on Its Mechanism}

\author{
Tang, $\operatorname{Kan}^{a}$ \\ Tao, Huijin* $*, b, d$ \\ Jiang, Xinyu ${ }^{c}$ \\ ( ${ }^{a}$ School of Material Science and Engineering, Central South University, Changsha 410083) \\ $\left({ }^{b}\right.$ Key Lab of Nonferrous Materials Science and Engineering, Ministry of Education, Central South University, \\ Changsha 410083) \\ ( ${ }^{c}$ School of Chemistry and Chemical Engineering, Central South University, Changsha 410083) \\ ( ${ }^{d}$ State Key Laboratory For Power Metallurgy, Central South University, Changsha 410083)
}

\begin{abstract}
The reaction between titanyl hydroxy and titanium(IV) has long been interested in and studied as its value in catalysts, analytic chemistry and synthesis of novel ceramic materials. Nevertheless, the possible reaction mechanism and its final products have never been concluded under different experimental conditions. Even in some particular condition i.e. $\mathrm{pH}$ $<1$, different products had been proposed such as $\mathrm{Ti}\left(\mathrm{O}_{2}\right)^{2+}$ and $\mathrm{Ti}\left(\mathrm{O}_{2}\right)(\mathrm{OH})_{n-2}^{(4-n)+}$. In this article, a possible reaction mechanism has been proposed based on first-principles calculations in order to explain its experimental phenomenon under specific condition $(\mathrm{s})(\mathrm{pH}<1)$. The calculation is carried out by the combination of B3LYP functional and 6-311G $+(3 \mathrm{df}, 2 \mathrm{p})$ basis set. As to estimate the solvent effect, the IEF-PCM model was also introduced in the calculation. Based on the calculation level mentioned above, the reaction profile and heat were figured out, and heat of reaction is comparable to the experimental data $(27 \mathrm{~kJ} / \mathrm{mol}$ vs. $24 \mathrm{~kJ} / \mathrm{mol})$. The rate limiting steps are also identified by checking the transformation of molecular orbitals before and after triatomic ring annulation reaction and hydrogen $\left(\mathrm{H}^{+}\right)$transfer reaction. In the annulation reaction, the complicated interaction between Ti's $3 \mathrm{~d}$ orbitals and O's $2 \mathrm{p}$ orbitals acts crucial role in the transformation of bonding types into triatomic ring. In the hydrogen $\left(\mathrm{H}^{+}\right)$transfer reaction, the vacant orbital $1 \mathrm{~s}$ of hydrogen $\left(\mathrm{H}^{+}\right)$attacks the HOMO of the titanyl peroxo complex to form new $\mathrm{O}-\mathrm{H}$ bond while breaking the old $\mathrm{O}-\mathrm{H}$ bond in the complex. The two steps reaction model fits well with pseudo-first-order hypothesis of the general dynamics of the reaction and the deviation of the general reaction dynamics as well, which supports the thermochemistry results made by first principles calculation. We hope this possible mechanism not only explains the reaction products under the specific condition, but also can be extended to other transition metals' reactions with peroxo complex and find its value in the synthesis of epoxidation catalysts and ceramics.
\end{abstract}

Keywords titanium(IV); hydrogen peroxide; B3LYP; molecular orbital; reaction dynamics

\footnotetext{
*E-mail: thj@csu.edu.cn

Received May 7, 2012; published August 7, 2012.

Supporting information for this article is available free of charge via the Internet at http: //sioc-journal. cn.

Project supported by the National Natural Science Foundation of China (No. 50871124), the Open-End Fund for the Valuable and Precision Instruments of Central South University (CSUZC2012023) and the Open Fund of State Key Laboratory of Powder Metallurgy.

项目受国家自然科学基金(No. 50871124)、中南大学贵重仪器使用开放基金(CSUZC2012023)和中南大学粉末冶金国家重点实验室开放基金资助.
} 


\section{1 引言}

正钛酸钛(IV)中心与过氧化氢的溶液反应很早就已 经开始被加以关注和研究 ${ }^{[1]}$. 该反应在分析化学 ${ }^{[2,3]}$ 、均 相 ${ }^{[4,5]} /$ 非均相 ${ }^{[6,7]}$ 环氧化催化剂和精细陶瓷材料 ${ }^{[8 \sim 11]}$ 的制 备中均有重要的用途. 不同的 $\mathrm{pH}$ 条件下, 钛中心过氧 化物在溶液中的离子形式和显现出的颜色十分复杂, 在 一定范围内, 随着 $\mathrm{pH}$ 的升高 $(\mathrm{pH}<1 \sim \mathrm{pH}>10)$, 溶液逐 渐经历橙红-黄绿-无色的变化 ${ }^{[12,13]}$. Schwarzenbach ${ }^{[12]}$ 通 过多种实验手段(电化学、单晶结构分析)系统地研究了 不同 $\mathrm{pH}$ 条件下该反应可能的产物形式并指出钛中心离 子与过氧化氢结合的主要特征是形成钛的 $\eta^{2}$ 型过氧根 配体. 然而之后的研究对于这一反应仍无定论, 不同的 实验现象依然存在, 如在 $\mathrm{pH}>10$ 的条件下, 黄绿色溶 液并未转变为无色 ${ }^{[14]}$.

鉴于在较大 $\mathrm{pH}$ 范围内钛中心与过氧化氢反应的复 杂性, 本文主要考察在酸性条件下该反应的机理与可能 产物. 然而即使在简化了的 $\mathrm{pH}<1$ 的酸性条件下, 关于 反应产物的争论 ${ }^{[12,15,16]}$ 依然存在. Schwarzenbach 通过电 化学法考察假想反应 $\mathrm{Ti}^{4+}+\mathrm{H}_{2} \mathrm{O}_{2} \rightarrow \mathrm{Ti}\left(\mathrm{O}_{2}\right)(\mathrm{OH})_{n-2}^{(4-n)+}+$ $n \mathrm{H}^{+}$, 得出 $n=3$ 从而获得产物为 $\left[\mathrm{Ti}\left(\mathrm{O}_{2}\right) \mathrm{OH}\right]^{+}$的猜测 ${ }^{[12]}$. Rotzinger 通过离子交换色谱法研究假想反应 $\mathrm{TiO}^{2+}+$ $\mathrm{H}_{2} \mathrm{O}_{2} \leftrightarrow \mathrm{Ti}\left(\mathrm{O}_{2}\right)^{2+}+\mathrm{H}_{2} \mathrm{O}$, 得出产物为 $\mathrm{Ti}\left(\mathrm{O}_{2}\right)^{2+}$ 的猜 测 ${ }^{[15]}$. 本文计划通过第一性原理计算, 寻找该反应可能 的反应路径并试探找出最终的产物, 为这一问题提供理 论上的解释, 以便对比验证实验的结果, 为今后该反应 合理利用于实际提供指导.

\section{2 计算方法和细节}

所有的计算均在 Gaussian $03 \mathrm{~W}$ 程序内完成 ${ }^{[17]}$. 初 始的反应物为 $(\mathrm{Ti}=\mathrm{O})^{2+}$ 和 $\mathrm{H}_{2} \mathrm{O}_{2}$. 其中钛中心被定义为 $(\mathrm{Ti}=\mathrm{O})^{2+}$ 是考虑到 $\mathrm{pH}<1$ 的条件下, 该结构已被光谱 实验数据所证实 ${ }^{[18,19]}$. $\mathrm{H}_{2} \mathrm{O}_{2}$ 尽管是一种结构非常简单的 分子, 但要获得精确可靠的计算结果往往使用一些带有 极化弥散函数的高等级基组 ${ }^{[20]}$. 我们测试了一些特定 的泛函和基组的组合并与已有的计算和实验结果进行 了比较(如表 1 所示). 非常明显的, PW91 基组不适合用
于含过氧化氢体系的计算, 因其在双氧水各项结构参数 与实际数据的较大偏差, 尤其是二面角方面, 与实验值 的误差超过 30\%. 而本文拟采用的 B3LYP 6-311G+ $(3 \mathrm{df}, 2 \mathrm{p})$ 在几乎所有的几何参数的计算方面都表现良好, 尤其是在二面角和 $\mathrm{O}-\mathrm{H}$ 键长方面，总体表现超过 MP2 算法和更高等级的 Post-Hartree Fock 算法, 因此本文在 密度泛函 DFT 水平上进行的计算使用 Beck's 杂化交换 泛函 ${ }^{[21]}$ 和 LYP 关联泛函 ${ }^{[22]}$ (B3LYP). B3LYP 泛函如前所 述在本文计算体系下精度可靠, 且效率较高, 被大量用 于类似体系的计算 ${ }^{[23 ~ 26]}$. 通过表 1 数据的对比, 弥散函 数的不断加入可以显著提高计算的精度, 因此结合计算 精度要求和溶剂效应方面的考虑, 选用 Pople 三重䢃裂 价键极化基组 6-311G+, 并对 $\mathrm{Ti}$ 原子增加三个 $\mathrm{d}$ 型弥 散轨道和一个 $\mathrm{f}$ 型弥散轨道, 对 $\mathrm{C}, \mathrm{N}, \mathrm{O}$ 原子分别增加两 个 $\mathrm{p}$ 型弥散轨道. 过渡态搜索一般情况下使用标准 QST2 和 QST3 方法, 且对所有过渡态都考察其 Hessian 矩阵验证其具有唯一的反映反应路径的虚频并使用 IRC 算法验证反应路径. 本文使用 IEF (integral equation formalism)极化连续溶剂模型 ${ }^{[27]}$ 对水的溶剂效应进行计 算. 大多数结构在溶剂模型中的收玫十分困难, 为了加 快收玫速度和降低收玫难度, 涉及到的多数在溶剂中的 优化采用 gdiis $^{[28]}$ 算法, 且只对非过渡态结构进行溶剂 模型下的优化. 反应物、过渡态、中间产物、最终产物 的能量参考文献中的评估方式 ${ }^{[4]}$, 即 $E_{\mathrm{cal}}=E_{\mathrm{total}}(0 \mathrm{~K})+$

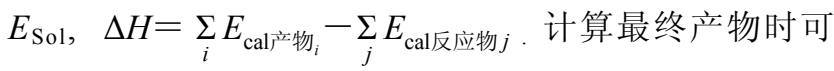
能涉及弱相互作用, 因此进行了 BSSE 校正 ${ }^{[29]}$, 结果显 示该处两个分子碎片的基组重叠并不明显, 对结合能的 影响不超过 0.001 Hartree. 参考文献 ${ }^{[25]}$, 计算过程中如 果涉及到过氧化氢的三重态, 将会引起体系能量计算结 果的显著上升，且体系波函数具有 $90 \%$ 的基态 $\mathrm{HF}$ 特征， 为了减少计算开销, 本文的电子体系被设定为严格闭壳 层体系, 即自选多重度始终为 1 . 所有的结构优化都不 加入任何对称性限制, 优化计算的收玫标准为 Maximum Force $\leqslant 0.000450$, RMS Force $\leqslant 0.000300$, Maximum Displacement $\leqslant 0.001800$, RMS Displacement $\leqslant 0.001200$.

表 1 不同计算方法得到的双氧水 $\left(\mathrm{H}_{2} \mathrm{O}_{2}\right)$ 分子的几何结构参数

Table 1 Structural parameters deduced by various of computation methods

\begin{tabular}{|c|c|c|c|c|}
\hline Computation method & Length of $\mathrm{O}-\mathrm{O}$ bond $/ \AA \AA$ & Angle of $\mathrm{O}-\mathrm{O}-\mathrm{H} /$ degree & Dihedral angle/degree & Length of $\mathrm{O}-\mathrm{H}$ bond $/ \AA$ \\
\hline MP2 6-31G(3df) & 1.439 & 100.3 & 109.2 & 0.969 \\
\hline MP2 6-31G(d) $)^{[30]}$ & 1.468 & 98.7 & 121.2 & 0.976 \\
\hline B3LYP 6-31G(3df) & 1.441 & 101.0 & 109.3 & 0.969 \\
\hline B3LYP $6-311 \mathrm{G}++(3 \mathrm{df}, 3 \mathrm{pd})^{[31]}$ & 1.446 & 100.8 & 112.5 & 0.966 \\
\hline B3LYP $6-311 \mathrm{G}+(3 \mathrm{df}, 2 \mathrm{p})^{a}$ & 1.446 & 100.9 & 111.9 & 0.965 \\
\hline $\operatorname{CCSD}(\mathrm{T}) \mathrm{CC}-\mathrm{PCVTZ} \mathrm{Z}^{[32]}$ & 1.455 & 99.6 & 113.9 & 0.963 \\
\hline B3PW91 6-311G+ & 1.506 & 99.6 & 144.2 & 0.978 \\
\hline Experimental data $^{[33,34]}$ & 1.464 & 99.4 & 111.8 & 0.965 \\
\hline
\end{tabular}

采本文采用的算法, 黑色字体表示与实验值符合较好的结果. 


\section{3 结果与讨论}

\section{1 反应路径与反应热形成的微观解释}

\section{1 .1 不同时期反应体系的分子结构与反应热的生成}

正钛酸钛中心与双氧水反应首先被考虑为两个反 应物分子 $(\mathrm{Ti}=\mathrm{O})^{2+}$ 和 $\mathrm{H}_{2} \mathrm{O}_{2}$ 由无穷远的能量参考零点 $(\mathrm{O})$ 如图 1(1), (2)依靠弱成键作用自发靠近而相互接触的过 程, 形成反应络合物 $\mathrm{A}$, 其主要结构和键长参数如图 1(3)所示. 反应络合物 A 经过过渡态结构 TS1 到达中间 产物 B. 过渡态 TS1 和中间产物 B 的结构如图 1(4), (5) 所示, 过渡态具有唯一的虚频- $169.39 \mathrm{~cm}^{-1}$. 通过计算 可知，反应络合物 $\mathrm{A}$ 与过渡态 $\mathrm{TS} 1$ 之间的反应能垒约为 $8 \mathrm{~kJ} / \mathrm{mol}(0 \mathrm{~K}, \mathrm{Vacuum})$. 这一反应对于形成特定的钛氧 三元环十分重要, 故反应络合物 $\mathrm{A}$ 与中间产物 $\mathrm{B}$ 这一过 程中具体成键及相应分子轨道的变化将在 3.1.2 节中进 行分析.

反应中间产物 $\mathrm{B}$ 此后会发生 $\mathrm{H}^{+}$转移反应. 基于两 个过氧根与钛中心形成的三元环之间的 $\sigma_{\mathrm{s}}$ 镜面对称性 的考虑, 无论 $\alpha-\mathrm{H}$ 或是 $\beta-\mathrm{H}$ 的转移反应都将生成唯一的 产物, 如图 1(8)所示. 然而实际的计算结果是该 $\mathrm{H}$ 转移 反应伴随着 $\sigma_{\mathrm{s}}$ 对称性的失去, 进而形成了两种具有结构 和能量差异的 $\mathrm{H}$ 转移反应产物 $\mathrm{C}^{\prime}$ 与 $\mathrm{C}$, 分别如图 1(6)(8) 所示, 其中我们定义能量较低的产物结构为 $\mathrm{C}$ 并规定其 上过氧根连接的氢离子为 $\alpha$ 氢离子. 在这两种结构之间, 存在一个过渡态 (虚频为 $-1756.96 \mathrm{~cm}^{-1}$ ), 该结构具有 一个处于 $\alpha$ 氧和 $\beta$ 氧之间的 $\mathrm{H}$ 原子, 生成了一个 150 $\mathrm{kJ} / \mathrm{mol}$ 的反应能垒 $(0 \mathrm{~K}, \mathrm{Vacuum})$, 因此我们倾向于认为 在常温条件下 $\mathrm{C}^{\prime}$ 与 $\mathrm{C}$ 的结构同时存在. $\mathrm{H}^{+}$转移反应再次 发生, 以使得初始 $\mathrm{H}_{2} \mathrm{O}_{2}$ 结构中的两个氢原子都转移至 钛酸根的氧原子上形成类似 $\mathrm{H}-\mathrm{O}-\mathrm{H}$ 的结构. 这一步 反应使得分子体系重新恢复了两个三元环之间的 $\sigma_{\mathrm{s}}$ 镜 面对称性, 即最终产物 D 所具有的对称性. 计算的结果 接近 Rotzinger 的实验猜测 ${ }^{[15]}$, 然而将孤立的 $\mathrm{Ti}\left(\mathrm{O}_{2}\right)^{2+}$ 与 $\mathrm{H}_{2} \mathrm{O}$ 结构优化后的能量相加, 无论是在真空还是溶剂模 型的计算环境下, 都明显高于相应条件下产物 D 的能 量, 故我们倾向认为 $\mathrm{Ti}$ 与类似水分子结构的成键联系 不能轻易忽略, 最终产物的形式应符合 $\mathrm{Ti}\left(\mathrm{O}_{2}\right)^{2+} \cdot \mathrm{H}_{2} \mathrm{O}$ 的形式. 以上两步反应是整个反应的关键步骤, 具体的 机理讨论和成键形式将在 3.1.3 和 3.2 节予以讨论. 以下 我们列出总反应涉及到的各步反应:

$$
\mathrm{TiO}^{2+}+\mathrm{H}_{2} \mathrm{O}_{2}=\mathrm{TiO}^{2+} \cdot \mathrm{H}_{2} \mathrm{O}_{2}
$$

$$
\mathrm{TiO}^{2+} \cdot \mathrm{H}_{2} \mathrm{O}_{2}=\mathrm{HO}-\mathrm{Ti}-\mathrm{OOH}
$$

$$
\mathrm{HO}-\mathrm{Ti}-\mathrm{OOH}+\mathrm{H}^{+}=\mathrm{Ti}\left(\mathrm{O}_{2}\right)^{2+} \cdot \mathrm{H}_{2} \mathrm{O}+\mathrm{H}^{+}
$$

$$
\begin{aligned}
& \begin{array}{cccc}
\left(\mathrm{Ti}=\mathrm{O}^{2+}\right)^{2+} \\
1.515 \\
1
\end{array}
\end{aligned}
$$

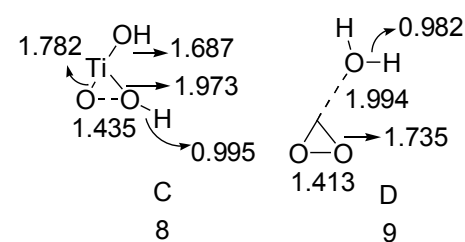

图 1 反应物、过渡态、中间产物和产物的几何构型和主要结构参数 (键长单位为 $\AA$ )

Figure 1 The geometry configuration and main constants of the reactants, transition states, intermediates and product (bond length in $\AA$ )

不同时期的反应步骤都伴随着体系能量的降低，与 实验中这一反应为放热反应的结论相符. $0 \mathrm{~K}$ 下的不同 时期分子结构真空中的能量和添加溶剂模型后的能量 在表 2 中予以汇总. 从中不难发现, 添加溶剂模型与否 并不影响反应热的变化趋势, 然而溶剂模型的加入有效 地考虑了不同情况下分子结构中引入的 $\mathrm{H}^{+}$受到的溶剂 稳定化效果, 进而使得反应热数据更加接近实验值. 使 用考虑溶剂效应的反应热数据，我们绘制了该反应的反 应路径图(如图 2 所示), 其中如前所述受到计算资源的 限制, 过渡态的几何全优化并未在溶剂模型中进行, 因 此过渡态的能量并未严格按照比例进行绘制. 最终我们 得出总反应热为 $27.55 \mathrm{~kJ} / \mathrm{mol}$. 就我们所知, 并无直接 的反应热数据可得, 而该反应在特定条件下 $\left(25^{\circ} \mathrm{C}, 10^{5}\right.$ $\mathrm{Pa}$ ) 的反应平衡常数约为 $8700 \mathrm{~L} / \mathrm{mol}$, 代入方程 $\Delta G=-R T \ln K$, 可得 $24 \mathrm{~kJ} / \mathrm{mol}$ 左右的反应自由能变,

\begin{tabular}{|c|c|c|c|c|c|c|c|c|c|}
\hline \multirow{2}{*}{ 计算模型 } & \multicolumn{9}{|c|}{ 不同时期的结构的能量/Hartree } \\
\hline & $\mathrm{O}$ & A & TS1 & $\mathrm{B}$ & $\mathrm{C}^{\prime}$ & TS2 & $\mathrm{C}$ & $\mathrm{D}$ & $\mathrm{Ti}\left(\mathrm{O}_{2}\right)^{2+}+\mathrm{H}_{2} \mathrm{O}$ \\
\hline Vacuum & -1075.4585 & -1075.5969 & -1075.5938 & -1075.6198 & -1075.6028 & -1075.5633 & -1075.6234 & -1075.6284 & -1075.4912 \\
\hline IEFPCM & -1076.1656 & -1076.1700 & - & -1076.1711 & -1076.1716 & - & -1076.1739 & $-1076.1760^{a}$ & -1075.1555 \\
\hline
\end{tabular}
该自由能变与计算得出的反应热的结果较为接近.

表 2 不同时期分子结构真空中的能量和添加溶剂模型后的能量

Table 2 Results of molecular total energy in vacuum and by applying solvent model

${ }^{a}$ 此处在能量中计入了氢键的能量, 氢键的能量参考文献[35], 为 $3.2 \mathrm{cal} / \mathrm{mol}$. 


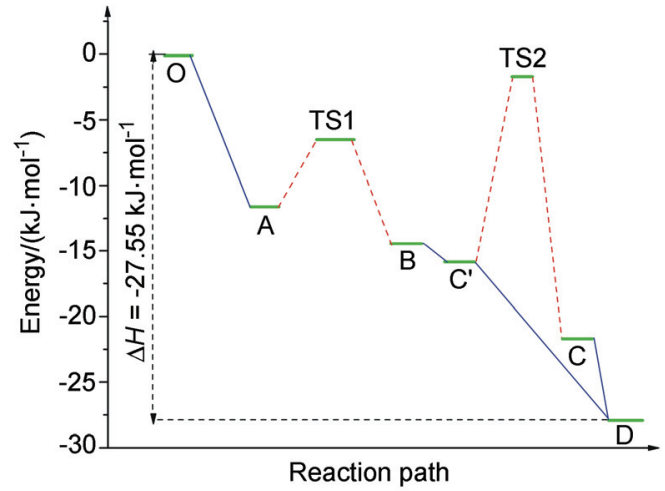

图 2 反应路径-能量关系图

Figure 2 Reaction profile (path vs. energy)

\subsection{2 钛氧三元环的形成机理}

如前所述, 两个反应物分子 $(\mathrm{Ti}=\mathrm{O})^{2+}$ 和 $\mathrm{H}_{2} \mathrm{O}_{2}$ 由无 穷远自发靠近而相互接触形成反应络合物 $\mathrm{A}$ 后, 会通过 一个具有较小激活能 $(8 \mathrm{~kJ} / \mathrm{mol})$ 的能垒进而形成钛氧三元 环结构. 因此钛中心和过氧根中心的成键作用在反应前 后的变化是一个关键的问题. 通过对计算得出的分子轨 道信息的笁选, 在反应前后能量体现出明显变化并对成 键贡献明显的分子轨道汇总于表 3. 从轨道电子云的特 征分析(如图 3), 第 19 号轨道在反应前只具有钛酸根中 $\mathrm{Ti}$ 的 $3 \mathrm{~d}_{z}^{2}$ 和 $\mathrm{O}$ 的 $2 \mathrm{p}_{z}$ 的成键作用, 然而在反应后铁酸根 上 $\mathrm{Ti}$ 的 $3 \mathrm{~d}_{z}{ }^{2}$ 和 $\mathrm{O}$ 的 $2 \mathrm{p}_{z}$ 发生了较大扭曲, 与过氧根的 $2 \mathrm{p}$ 轨道之间均有作用, 形成了一个较为复杂的 $\mathrm{p}-\mathrm{d}-\mathrm{p}$ 的成 键模式. 第 20 号轨道反应前的 $\mathrm{Ti}$ 的 $3 \mathrm{~d}_{y z}$ 的过氧根氧的 $2 \mathrm{p}$ 轨道并无成键作用, 反应后 $3 \mathrm{~d}_{y z}$ 与过氧根 $\mathrm{p}$ 电子的成 键变得十分明显. 第 21 号轨道反应具有较弱的 $3 \mathrm{~d}_{x y}$ 与过 氧根的成键效应, 反应后该效应明显增强. 第 22 号轨道 的明显特征在于 $\mathrm{Ti}$ 从与过氧根中的一个氧以 $3 \mathrm{~d}_{x z}-2 \mathrm{p}$ 方 式成键变为与过氧根的两个氧以同样方式成键, 与反应 理想中应具有动力学模式符合的很好. 有趣的是, HOMO 即第 23 号轨道反应前铁酸根上的氧与过氧根之 间形成了一个 $2 p-2 p$ 的键连, 在反应后这一键连方式减 弱至接近消失.

表 3 成环反应 $\mathrm{A} \rightarrow \mathrm{B}$ 前后电子云产生显著变化的分子轨道

Table 3 The list of molecular orbitals with distinguished change of electrons' distribution before and after reaction $\mathrm{A} \rightarrow \mathrm{B}$

\begin{tabular}{ccccc}
\hline \multirow{2}{*}{ 轨道序号 $^{a}$} & \multicolumn{4}{c}{ 不同结构能量/Hartree } \\
\cline { 2 - 5 } & $E_{\mathrm{A}}$ & $E_{\mathrm{TS} 1}$ & $E_{\mathrm{B}}$ & $\left(E_{\mathrm{B}}-E_{\mathrm{A}}\right) / E_{\mathrm{A}}$ \\
\hline 23 & -0.357 & -0.749 & -0.825 & 1.10 \\
22 & -0.388 & -0.796 & -0.832 & 1.14 \\
21 & -0.403 & -0.847 & -0.838 & 1.08 \\
20 & -0.404 & -0.850 & -0.847 & 1.10 \\
19 & -0.412 & -0.853 & -0.855 & 1.08 \\
\hline
\end{tabular}

${ }^{a} \mathrm{HOMO}($ 最高占轨)即 23 号轨道, 其余轨道依次递减.
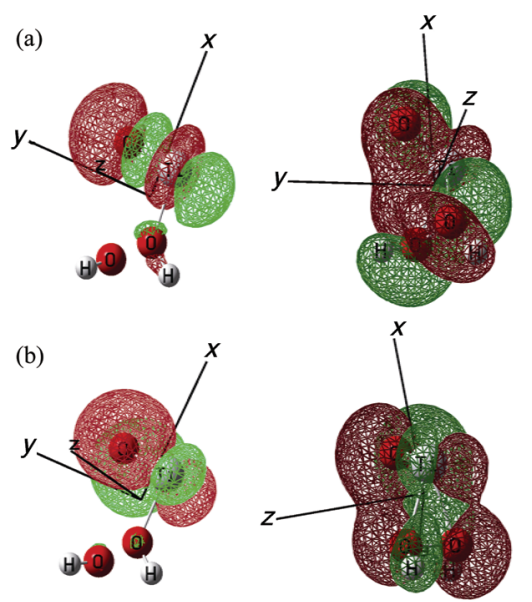

(c)
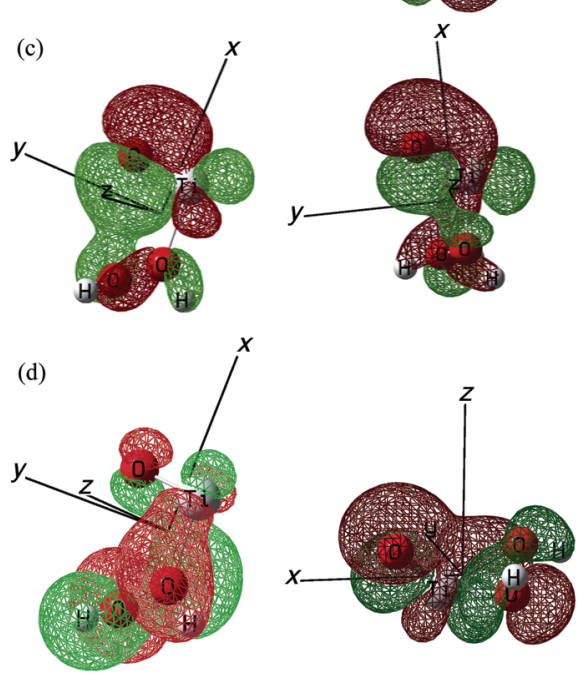

(e)

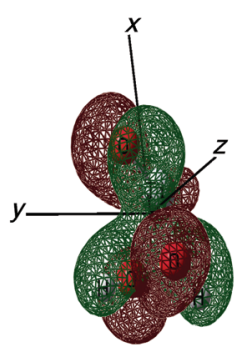

图 3 钛氧三元环在反应前后的成键模式 $(a \sim e)$, 其中电子云等值面 均为 $0.02 \mathrm{e} / \AA^{3}$

Figure 3 The bonding modes between Ti and $\eta^{2}$ peroxo group ligand, the electrons' isosurface value is set to be $0.02 \mathrm{e} / \AA^{3}$

(a) HOMO-4(p-d) $\rightarrow$ (p-d-p); (b) HOMO-3 none $\rightarrow$ (d-p); (c) HOMO-2 weak $(d-p) \rightarrow$ strong $(d-p)$; (d) HOMO-1 single side $(d-p) \rightarrow$ double sides $(d-p) ;(e)$ HOMO strong $(p-p) \rightarrow$ weak $(p-p)$

\subsection{3 氢 $\left(\mathrm{H}^{+}\right)$转移反应}

如前文所述, 我们认为 $\mathrm{A} \rightarrow \mathrm{B}$ 与 $\mathrm{C}\left(\mathrm{C}^{\prime}\right) \rightarrow \mathrm{D}$ 的两步反 应是在全部反应路径中较为重要的部分, 为了不至于混 淆, 在此我们对于反应 $\mathrm{C}\left(\mathrm{C}^{\prime}\right) \rightarrow \mathrm{D}$ 单独加以讨论. 考虑到 $\mathrm{C}$ 与 $\mathrm{C}^{\prime}$ 结构的相似性，以下所有涉及到该步反应的讨论 都只对中间产物 $\mathrm{C}$ 进行. 首先, 假定溶液环境中的氢离 子靠近具有 $\alpha 、 \beta$ 氢原子的中间产物 $\mathrm{B}$ (为了体现 $\mathrm{O}-\mathrm{H}$ 在初始分子轨道中的位置, 仅有此处的电子云密度设定 
为 $0.15 \mathrm{e} / \AA^{3}$, 其余结构均为 $\left.0.02 \mathrm{e} / \AA^{3}\right)$, 并与其 HOMO 轨道接触, 经过约为 $400 \mathrm{~kJ} / \mathrm{mol}$ (Vacuum, $0 \mathrm{~K}$ )的能垒, 到达过渡态 TS3 (虚频为 $-853.77 \mathrm{~cm}^{-1}$ ). 考虑到未引入 溶剂模型, 溶剂对于过渡态中松散 $\mathrm{H}^{+}$离子的稳定化作 用被低估, 因此反应能垒可能被大大高估. 对于溶剂模 型中质子稳定化的准确计算, 不同的模型(如 IEFPCM 或 SMD)往往会有很大的偏差, 有时会得出相反的结 论 $^{[4]}$, 因此这仍将是今后需要进一步加以计算的内容. $\mathrm{TS} 3$ 中新加入的氢离子与钛酸根上的氧原子产生 $\mathrm{O}-\mathrm{H}$ 成键效应, 同时大大削弱了 $\beta$ 氢与过氧根氧原子的联系 (如图 4(a)所示). 反应经过过渡态, 完成新 $\mathrm{O}-\mathrm{H}$ 键的生 成和旧 $\mathrm{O}-\mathrm{H}$ 键的脱去. 反应前后产生明显变化并对体 系成绩有影响的分子轨道汇总于表 4 , 有趣的是氢转移 反应对总体系能量的降低并无显著贡献, 对降低体系总 能具有明显贡献的是 14 号和 20 号分子轨道, C 与 D 结 构的 14 号和 20 号分子轨道如图 5 所示, 其中 14 号轨道 的氧原子的 $\mathrm{p}$ 轨道稳定了新加入的氢离子, 20 号轨道增 强了过氧根两个氧原子的连接和铁酸根中钛氧间的连 接.

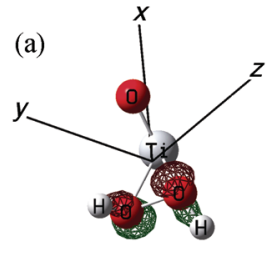

B

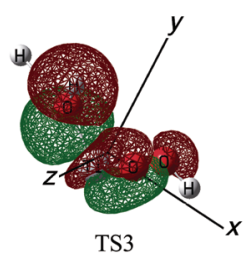

TS3

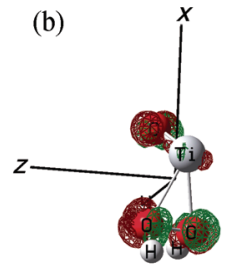

$\mathrm{B}$

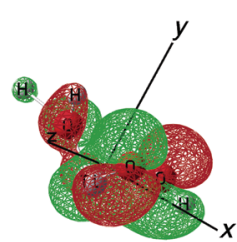

TS3
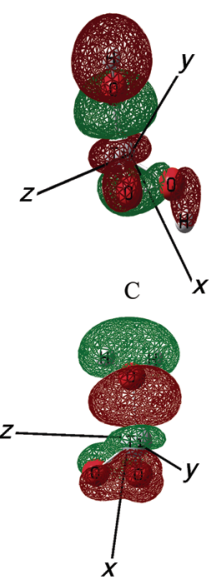

$\mathrm{D}$
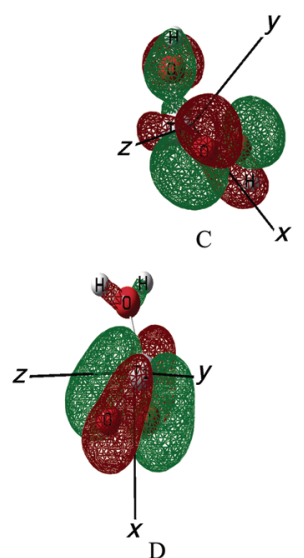

图 4 氢转移反应过程中第 17 号分子轨道(a)和第 23 号分子轨道(b) HOMO 的变化

Figure 4 The transformation of HOMO (a) and HOMO-6 (b) during $\mathrm{H}^{+}$-transfer reaction
表 4 氢转移反应 $\mathrm{B} \rightarrow \mathrm{C} \rightarrow \mathrm{D}$ 前后电子云产生显著变化的分子轨道

Table 4 The list of molecular orbitals with distinguished change of electrons' distribution before and after $\mathrm{H}^{+}$-transfer reaction $\mathrm{B} \rightarrow \mathrm{C} \rightarrow \mathrm{D}$

\begin{tabular}{cccccc}
\hline \multirow{2}{*}{ 轨道序号 } & \multicolumn{5}{c}{ 不同结构能量/Hartree } \\
\cline { 2 - 6 } & $E_{\mathrm{B}}$ & $E_{\mathrm{C}}$ & $E_{\mathrm{D}}$ & $\left(E_{\mathrm{B}}-E_{\mathrm{C}}\right) / E_{B}$ & $\left(E_{\mathrm{C}}-E_{\mathrm{D}}\right) / E_{\mathrm{C}}$ \\
\hline $23^{a}$ & -0.825 & -0.779 & -0.766 & $5.8 \%$ & $1.7 \%$ \\
20 & -0.847 & -0.855 & -0.923 & $-0.9 \%$ & $-8.0 \%$ \\
17 & -1.010 & -1.000 & -0.974 & $0.9 \%$ & $2.6 \%$ \\
14 & -1.414 & -1.455 & -1.528 & $-2.9 \%$ & $-5.0 \%$ \\
\hline
\end{tabular}

${ }^{a} \mathrm{HOMO}($ 最高占轨)即 23 号轨道, 其余轨道依次递减.
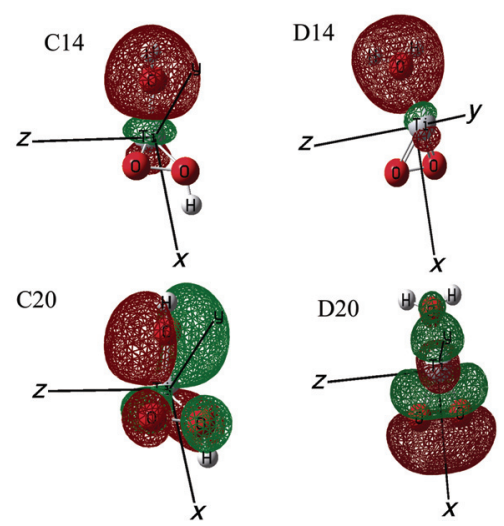

图 $5 \mathrm{C}$ 与 $\mathrm{D}$ 的 14 号和 20 号轨道在氢转移反应前后的电子云分布(电 子云等值面均为 $0.02 \mathrm{e} / \AA^{3}$ )

Figure 5 The possibility distribution of electrons in HOMO-9 and HOMO-3 before and after the $\mathrm{H}^{+}$-transfer reaction from $\mathrm{C}$ to $\mathrm{D}$ (the electrons' isosurface value is set to be $0.02 \mathrm{e} / \AA^{3}$ )

\section{2 反应机理与宏观反应动力学的联系}

在之前类似体系的计算中许多不同的反应机理被 反复的提出, 如分子内脱水 ${ }^{[4]}$ 、分子间氢转移 ${ }^{[2]}$ 等. 由于 各自计算体系的不同, 不同的反应机理确实有可能在不 同的情形下分别存在. 然而之前的计算尽管在热力学上 都是可行的，但并没有同各自情况下的反应动力学作对 比和尝试性的解释，这在一定程度上影响了计算的意义 和可信度. 部分实验已经证实本文研究的这一反应在反 应物浓度较小时是一个 “准一级” 反应，而在浓度较高 时偏离一级近似 ${ }^{[16,36]}$. 成环反应较为平缓的势能面可以 解释当反应物浓度较小时, 该二级反应体现出准一级反 应的特性控制反应的速率. 当反应物浓度上升后, 氢转 移反应开始影响反应动力学进而使其偏离准一级近似 的结果. 基于上述分析, 我们试图通过两步单向连串反 应模型解释该反应的动力学现象. 假定反应 $\mathrm{A} \rightarrow \mathrm{B}$ 与 $\mathrm{C} \rightarrow \mathrm{D}$ 的反应速率常数分别为 $k_{1}, k_{2}$, 对于反应物 $\mathrm{A}$ 和中 间产物 $\mathrm{C}$ 的浓度可以列出下列微分方程:

$$
\begin{aligned}
& \frac{\mathrm{d} C_{\mathrm{A}}}{\mathrm{d} t}=-k_{1} C_{\mathrm{A}} \\
& \frac{\mathrm{d} C_{\mathrm{C}}}{\mathrm{d} t}=k_{1} C_{\mathrm{A}, 0} \exp \left(-k_{1} t\right)-k_{2} C_{\mathrm{C}}
\end{aligned}
$$

解微分方程(2), 结合边界条件 $t=0, C_{\mathrm{B}} \approx 0, C_{\mathrm{A}}=C_{\mathrm{A}, 0}$, 
得

$$
C_{\mathrm{c}}=\left(k_{1} C_{\mathrm{A}, 0} \mathrm{e}^{-k_{1} t}\right) /\left(k_{2}-k_{1}\right)-\left(k_{1} C_{\mathrm{A}, 0} \mathrm{e}^{-k_{2} t}\right) /\left(k_{2}-k_{1}\right)(3)
$$

由于 $C_{\mathrm{D}}=C_{\mathrm{A}, 0}-C_{\mathrm{A}}-C_{\mathrm{C}}$ (4), 将方程(1), (3)代入(4), 可 得

$$
C_{\mathrm{D}}=C_{\mathrm{A}, 0}\left[1-\left(k_{2} \mathrm{e}^{-k_{1} t}-k_{1} \mathrm{e}^{-k_{2} t}\right) /\left(k_{2}-k_{1}\right)\right]
$$

反应动力学方程(5)可以较为合理地解释动力学实 验中产物浓度对时间的变化规律, 通过不同条件下对参 数 $k_{1}, k_{2}$ 的拟合, 可以吸收诸如不同浓度和离子强度等 因素对于动力学的影响, 既可以在较低反应物浓度时显 现出一级反应的特性, 又能在较高反应物浓度时产生浓 度数值对时间的指数型收玫, 整体体现出近似幂指数衰 减的特性，与相关的实验事实完全相符 ${ }^{[16,36]}$. 然而不同 浓度或离子强度下的实际反应是比较复杂的, 浓度与时 间关系的定量实验的数据难以直接从已有实验文献中 获得, 给方程 (5)在不同条件下的参数拟合带来很大困 难, 而单纯直接使用这种幂指数组合的形式对反应速率

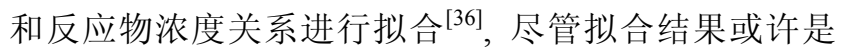
比较可观的, 我们认为是缺乏实际意义的, 因为这样的 做法与反应机理几乎毫无关联.

\section{4 结论}

长久以来, 溶液条件下, 钛 (IV)与过氧化氢的络合 物的具体存在形式和形成机理没有得到明确解释. 本文 回顾了不同 $\mathrm{pH}$ 尤其是酸性条件 $(\mathrm{pH}<1)$ 下不同实验检 测条件下对最终产物结构的争议, 并试图开辟一条可行 的计算途径进而解释反应机理和实验现象. 借助高等级 的弥散极化基组、表现良好的泛函以及 IEFPCM 溶剂模 型, 得出产物 $\mathrm{Ti}\left(\mathrm{O}_{2}\right)^{2+} \cdot \mathrm{H}_{2} \mathrm{O}$ 的反应热数据(约为 27 $\mathrm{kJ} / \mathrm{mol}$ ) 接近实验值, 从而获得了一条热力学可行的反应 路径. 在此基础上, 通过对于反应过渡态的计算, 得出 了 $\eta^{2}$ 型过氧根配体与钛中心结合的具体形式, 即不同的 全空 $3 \mathrm{~d}$ 轨道与过氧根的 $2 \mathrm{p}$ 电子产生的复杂的相互作用, 形成了钛氧三元环. 对反应过程中分子轨道的变化作进 一步的分析之后, 提出氢 $\left(\mathrm{H}^{+}\right)$转移反应假设. 但由于计 算资源的限制, 借助溶剂模型对氢转移反应能垒做更加 仔细的估算仍将是今后我们的主要工作. 成环反应和氢 转移反应假设合理地解释了宏观动力学的准一级反应 特征, 而且对于一般的情况也可以进行反应速率常数的 有效拟合. 然而对于这一反应的理论解释的意义不光局 限于此, 我们希望类似机理可以被用于解释其他具有全 空 $\mathrm{d}$ 轨道过渡族金属中心(如 $\mathrm{V}, \mathrm{Zr}, \mathrm{Nb}, \mathrm{Mo}$ 等 ${ }^{[4]}$ )与过氧 化物的反应中, 并为均相/非均相催化剂的设计和精细 陶瓷材料的制备等提供参考.

\section{References}

[1] Piccini, A. Z. Anorg. Chem. 1895, 10, 438.

[2] Takamura, K.; Matsumoto, T. Anal. Biol. Anal. Chem. 2008, 391, 951.
[3] Matsubara, C.; Kawamoto, N.; Takamura, K. Analyst 1992, 117, 1781.

[4] Antonova, N. S.; Carbo, J. J.; Kortz, U.; Kholdeeva, O. A.; Poblet, J. M. J. Am. Chem. Soc. 2010, 132, 7488.

[5] Kholdeeva, O. A. Top. Catal. 2006, 40, 229

[6] Bordiga, S.; Damin, A.; Bonino, F.; Ricchiardi, G.; Zecchina, A.; Tagliapietra, R.; Lamberti, C. Phys. Chem. Chem. Phys. 2003, 5, 4390.

[7] Ohno, T.; Masaki, Y.; Hirayama, S.; Matsumura, M. J. Catal. 2001, $204,163$.

[8] Liao, J. H.; Shi, L. Y.; Yuan, S. J. Phys. Chem. C 2009, 113, 18778.

[9] Ichinose, H.; Tearsaki, M.; Katsuki, H. J. Sol-Gel Sci. Technol. 2001, 22, 33 .

[10] Lee, K. H.; Song, S. W. ACS Appl. Mater. Interfaces 2011, 3, 3697.

[11] Niesen, T. P.; Bill, J.; Aldinger, F. Chem. Mater. 2001, 13, 1552.

[12] Muhlebach, J.; Muller, K.; Schwarzenbach, G. Inorg. Chem. 1970, 9, 2381.

[13] Mori, M.; Shibata. N.; Kyono, E.; Ito, S. Bull. Chem. Soc. Jpn. 1956, $29,904$.

[14] Gao, Y. F.; Masuda, Y.; Peng, Z. F.; Yonezawaand, T.; Koumoto, K. J. Mater. Chem. 2003, 13, 608.

[15] Rotzinger, F. P.; Gratzel, M. Inorg. Chem. 1987, 26, 3704.

[16] Orhanovic, M.; Wilkins, R. G. J. Am. Chem. Soc. 1967, 89, 278.

[17] Frisch, M. J.; Trucks, G. W.; Schlegel, H. B.; Scuseria, G. E.; Robb, M. A.; Cheeseman, J. R.; Montgomery, J. A. Jr.; Vreven, T.; Kudin, K. N.; Burant, J. C.; Millam, J. M.; Iyengar, S. S.; Tomasi, J.; Barone, V.; Mennucci, B.; Cossi, M.; Scalmani, G.; Rega, N.; Petersson, G. A.; Nakatsuji, H.; Hada, M.; Ehara, M.; Toyota, K.; Fukuda, R.; Hasegawa, J.; Ishida, M.; Nakajima, T.; Honda, Y.; Kitao, O.; Nakai, H.; Klene, M.; Li, X.; Knox, J. E.; Hratchian, H. P.; Cross, J. B.; Adamo, C.; Jaramillo, J.; Gomperts, R.; Stratmann, R. E.; Yazyev, O.; Austin, A. J.; Cammi, R.; Pomelli, C.; Ochterski, J. W.; Ayala, P. Y.; Morokuma, K.; Voth, G. A.; Salvador, P.; Dannenberg, J. J.; Zakrzewski, V. G.;. Dapprich, S.; Daniels, A. D.; Strain, M. C.; Farkas, O; Malick, D. K.; Rabuck, A. D.; Raghavachari, K.; Foresman, J. B.; Ortiz, J. V.; Cui, Q.; Baboul, A. G.; Clifford, S.; Cioslowski, J.; Stefanov, B. B.; Liu, G.; Liashenko, A.; Piskorz, P.; Komaromi, I.; Martin, R. L.; Fox, D. J.; Keith, T.; Al-Laham, M. A.; Peng, C. Y.; Nanayakkara, A.; Challacombe, M. W.; Gill, P. M.; Johnson, B.; Chen, W.; Wong, M. W.; Gonzalez, C.; Pople, J. A. Gaussian 03, Revision B. 01, Gaussian Inc., Pittsburgh PA, 2003.

[18] Ellis, J. D.; Thompson, G. A. K.; Sykes, A. G. Inorg. Chem. 1976, 15,3173 .

[19] Gratzel, M.; Rotzinger, F. P. Inorg. Chem. 1985, 24, 2321.

[20] Koput, J. Chem. Phys. Lett. 1995, 236, 516.

[21] Lee, C.; Yang, C.; Parr, R. G. Phys. Rev. B 1988, 37, 785.

[22] Becke, A. D. J. Chem. Phys. 1993, 98, 5648.

[23] Bordiga, S.; Bonino, F.; Damin, A. Phys. Chem. Chem. Phys. 2007, 9, 4854.

[24] Lundin, A.; Panas, I.; Ahlberga, E. Phys. Chem. Chem. Phys. 2007, 9, 5997.

[25] Stare, J.; Henson, N. J.; Eckert, J. J. Chem. Inf. Model. 2009, 49, 833.

[26] Lundin, A.; Panas, I.; Ahlberg, E. J. Phys. Chem. A 2007, 111, 9080.

[27] Cossi, M.; Barone, V.; Mennucci, B.; Tomasi, J. Chem. Phys. Lett. 1998, 286, 253.

[28] Csaszar, P.; Pulay, P. J. Mol. Struct. 1984, 114, 31.

[29] Boys, S. F.; Bernardi, F. Mol. Phys. 1970, 4, 553.

[30] Bach, R. D.; Ayala, P. Y.; Schlegel, H. B. J. Am. Chem. Soc. 1996, $118,12758$.

[31] Maciel, G. S.; Bitencourt, A. P.; Ragni, M.; Aquilanti, V. Chem. Phys. Lett. 2006, 432, 383.

[32] Koput, J.; Carter, S.; Handy, N. C. J. Phys. Chem. A 1998, 102, 6325 .

[33] Khachkuruzov, G. A.; Przhevalskii, I. N. Opt. Spectrosc. 1974, 36, 172.

[34] Koput, J. J. Mol. Spectrosc. 1986, 115, 438.

[35] Feyereisen, M. W.; Feller, D.; Dixon, D. A. J. Phys. Chem. 1996 100, 2993.

[36] Wick, P. K.; Kissner, R.; Koppenol, W. H. Inorg. Chem. 2004, 43, 4805.

[37] Merenyi, G.; Lind, J.; Czapski, G.; Goldstein, S. Inorg. Chem. 2003, 42,3796 . 